\title{
Flavonoids from Orostachys Japonicus A. Berger Induces Caspase-dependent Apoptosis at Least Partly through Activation of p38 MAPK Pathway in U937 Human Leukemic Cells
}

\author{
Won Sup Lee ${ }^{1 \& *}$, Jeong Won Yun ${ }^{1 \&}$, Arulkumar Nagappan ${ }^{1}$, Ji Hyun Jung ${ }^{1}$, Sang \\ Mi Yi ${ }^{1}$, Dong Hoon Kim ${ }^{2}$, Hye Jung Kim ${ }^{3}$, GonSup Kim ${ }^{4}$, Chung Ho Ryu ${ }^{5 \&}$, Sung \\ Chul Shin ${ }^{6}$, Soon Chan Hong ${ }^{7}$, Yung Hyun $\mathrm{Choi}^{8}$, Jin-Myung Jung9
}

\begin{abstract}
Background: Orostachys japonicus A. Berger (A. Berger) is commonly used as a folk remedy for cancer therapy. However, the mechanisms of its anti-cancer activity are poorly investigated in human cancer cells. In this study, we investigated whether flavonoids extracted from Orostachys japonicus A. Berger (FEOJ) might have anticancer effects in human leukemia cells, focusing on cell death mechanisms. Materials and Methods: U937 human leukemic cancer cells were used. Results: FEOJ induced apoptosis in a dose-dependent manner in human U937 cancer cells. Flow cytometry revealed significant accumulation of cells with sub-G1 DNA content at the concentrations of $200 \mu \mathrm{g} / \mathrm{mL}$ and $400 \mu \mathrm{g} / \mathrm{mL}$. FEOJ-induced apoptosis was caspase-dependent through loss of mitochondrial membrane potential (MMP, $\Delta \Psi \mathrm{m}$ ) in human U937 cancer cells, which might be associated with suppression of Bcl-2 and XIAP proteins. FEOJ induced the p38 MAPK signaling pathway, playing at least in part an important role in FEOJ-induced apoptosis. Conclusions: This study suggested that FEOJ may induce caspase-dependent apoptosis in human leukemic cells by regulating MMP $(\Delta \Psi \mathrm{m})$ through suppressing Bcl-2 and X-IAP. In addition, the results indicated that upstream $\mathrm{p38}$ MAPK signaling regulates the apoptotic effect of FEOJ. This study provides evidence that FEOJ might have anti-cancer potential for human leukemic cells.
\end{abstract}

Keywords: Orostachys japonicus A. Berger - flavonoids - U937 - apoptosis - p38 MAPK - leukemia cells

Asian Pac J Cancer Prev, 16 (2), 465-469

\section{Introduction}

Apoptosis is an active-energy requiring process (a type I programmed cell death) with a distinctive phenotype characterized by cytoplasmic shrinkage, blebbing of the plasma membrane, chromatin condensation, DNA degradation, and nuclear fragmentation (Kerr et al., 1972; Walker and Sikorska, 1997). Most of apoptosis occurs through two major pathways (intrinsic pathway - mitochondria-mediated apoptosis and extrinsic pathwaydeath receptor-mediated apoptosis) (Kumi-Diaka et al., 2004).

Despite enormous efforts to control cancer development, the cancer incidence is still increasing (Jung et al., 2014). Given the results, we obviously need a paradigm shift to reduce the cancer incidence. One of the pragmatic ways is chemoprevention because the dietary agents and some phytochemicals are reported to induce apoptosis or enhance anti-cancer effects without significant toxicities in the normal cells (Palasap et al., 2014). This safely showing or enhancing anti-cancer activity derived from the dietary agents or phytochemicals has drawn our interests to seek the possibility of controlling cancer with minimal toxicity. The extracts of Orostachys japonicus A. Berger (A. Berger) has been used as a folk remedy for cancer treatment. Orostachys japonicus $A$. Berger belongs to a member of the family Liliaceae, and has plentiful flavonoids, which may exhibit anti-cancer properties. However, few studies have been conducted regarding the anti-cancer effects of flavonoids extracted from lyophilized A. Berger (FEOJ) and the molecular mechanisms of the effects are poorly understood in human cancer cells. In addition, with the growth of ecological movements, natural products have become more popular

Departments of ${ }^{1}$ Internal Medicine, ${ }^{2}$ Emergency Medicine, ${ }^{3}$ Pharmacology, ${ }^{7}$ Surgery, ${ }^{9}$ Neurosurgery, Institute of Health Sciences, ${ }^{5}$ Division of Applied Life Science (BK 21 Program), Institute of Agriculture and Life Science, ${ }^{6}$ Department of Chemistry, Research Institute of Life Science, ${ }^{4}$ School of Veterinary Medicine, Gyeongsang National University School of Medicine, Jinju, ${ }^{8}$ Department of Biochemistry, Dongeui University College of Oriental Medicine, Busan, Korea ${ }^{\&}$ Equal contributors *For correspondence: lwshmo@hanmail.net,lwshmo@gshp.gsnu.ac.kr 
for the prevention or treatment of cancer.

With this background information, we assumed that FEOJ are major ingredients have the anti-cancer effects of the extracts of Orostachys japonicus A.Berger (A.Berger) that has been used as a folk remedy for cancer treatment. Therefore, we isolated and fully characterized FEOJ, and investigated the mechanisms of anticancer effects of FEOJ on human leukemic cells.

\section{Materials and Methods}

\section{Preparation of FEOJ}

FEOJ isolated from the plants of Orostachys japonicus A. Berger was a generous gift from Dr. S.C. Shin (Department of Chemistry, Gyeongsang National University, Korea). FEOJ were extracted with polar organic solvents followed by RP-chromatography. FEOJ contains 16 flavonoids; (1): Procyanidin dimer gallate isomer; (2): Procyanidin dimer gallate isomer (3): Epigallocatechin-3-gallate (4): Procyanidin dimer digallate isomer (5): Epicatechin (6): Quercetin 3-O-rhamnosyl-7O-glucoside (7): Epicatechin gallate (8): Kaempferol 3-O-rhamnosyl-7-O-glucoside(9): Procyanidin dimer digallate isomer (10): Myricetin 3-O-glucoside (11): Quercetin 3-O-glucoside(12): Kaempferol-3O-rutinoside(13): Quercetin 7-O-rhamnoside(14): Kaempferol dideoxyhexosylhexoside (15): N1,N5,N10tri-p-(E,E,E)-coumaroylspermidine (16): Kaempferol.

\section{Cells and reagents.}

U937 human leukemic cells from the American type culture collection (Rockville, MD, USA) were cultured in RPMI 1640 medium (Invitrogen Corp, Carlsbad, CA, USA) supplemented with $10 \%(\mathrm{v} / \mathrm{v})$ fetal bovine serum (FBS) (GIBCO BRL, Grand Island, NY, USA), 1 $\mathrm{mM}$ L-glutamine, $100 \mathrm{U} / \mathrm{mL}$ penicillin, and $100 \mu \mathrm{g} / \mathrm{mL}$ streptomycin at $37^{\circ} \mathrm{C}$ in a humidified atmosphere of $95 \%$ air and 5\% CO2. Antibodies against Bcl-2, Bid, inhibitors of apoptosis protein-1 (IAP-1), IAP-2, X-linked IAP (XIAP), pro-caspase 3, pro-caspase 8, and pro-caspase 9 were purchased from Santa Cruz Biotechnology (Santa Cruz, CA, USA). Antibody against poly (ADP-ribose) polymerase (PARP) was purchased from PharMingen (San Diego, CA, USA). Antibody against $\beta$-actin was from Sigma (Beverly, MA). Peroxidase-labeled donkey anti-rabbit and sheep anti-mouse immunoglobulin, and an enhanced chemiluminescence (ECL) kit were purchased from Amersham (Arlington Heights, IL). Caspase activity assay kits were purchased from $R \& D$ systems (Minneapolis, MN, USA). All other chemicals not specifically cited here were purchased from Sigma Chemical Co. (St. Louis, MO, USA).

\section{Cell viability}

The cells were seeded onto 24 -well plates at a concentration of $1 \times 10^{5}$ cells $/ \mathrm{ml}$, and then treated with the indicated concentration of FEOJ for 48 h. 3-(4, 5-dimethylthiazol-2-yl)-2,5-diphenyltetrazolium bromide (MTT, $0.5 \mathrm{mg} / \mathrm{ml}$ ) was subsequently added to each well. After additional $2 \mathrm{hr}$-incubation, $100 \mu \mathrm{L}$ of a solution containing $10 \%$ SDS (pH 4.8) plus $0.01 \mathrm{~N} \mathrm{HCl}$ was added to dissolve the crystals. The absorption values at $570 \mathrm{~nm}$ were determined with an enzyme-linked immunosorbent assay (ELISA) plate reader.

Flow cytometry analysis for measurement of sub-G1 phase

The cells were plated at a concentration of $2 \times 10^{5}$ cells/well in six-well plates. After treatment of FEOJ, the cells were collected, washed with cold PBS, and then centrifuged. The pellet was fixed in $75 \%(\mathrm{v} / \mathrm{v})$ ethanol at $4^{\circ} \mathrm{C}$ for $1 \mathrm{~h}$. The cells were washed once with PBS, and re-suspended in cold PI solution $(50 \mu \mathrm{g} / \mathrm{ml})$ containing RNase A $(0.1 \mathrm{mg} / \mathrm{ml})$ in PBS ( $\mathrm{pH} 7.4)$ for $30 \mathrm{~min}$ in the dark. The cellular DNA was then stained by applying $250 \mathrm{ml}$ of propidium iodide $(50 \mathrm{mg} / \mathrm{ml})$ for $30 \mathrm{~min}$ at room temperature. The stained cells were analyzed by fluorescent activated cell sorting (FACS) on a FACScan flow cytometer (Becton Dickinson, San Jose, CA) for relative DNA content based on red fluorescence.

\section{Western blot analysis}

The suspending cells were harvested and lysed in the lysis buffer. The cells were disrupted by sonication and extracted at $4^{\circ} \mathrm{C}$ for $30 \mathrm{~min}$. The protein concentrations were quantified using the BioRad protein assay (BioRad Lab.,Hercules, CA, U.S.A.). The proteins of the extracts were resolved by electrophoresis, electrotransferred to a polyvinylidene difluoride membrane (Millipore, Bedford, MA), and then the membrane was incubated with the primary antibodies followed by a conjugated secondary antibody to peroxidase. After washing, the membranes were developed by enhanced chemiluminescence.

\section{Caspase activity assay.}

Caspase activity was determined by a colorimetric assay according to the manufacturer's protocol in a kit for caspase activity (R\&D Systems, Minneapolis, Minnesota, USA). In brief, the cells were lysed in the supplied lysis buffer. The supernatants were collected and incubated with the supplied reaction buffer containing dithiothreitol and substrates at $37^{\circ} \mathrm{C}$. The reaction was measured by determining the change in absorbance at $405 \mathrm{~nm}$ using the microplate reader.

Mitochondrial membrane potential (MMP, $\Delta \Psi m)$ assay

The MMP $(\Delta \Psi \mathrm{m})$ in living cells was measured by flow cytometry with the lipophilic cationic probe JC-1, which is a ratiometric, dual-emission fluorescent dye. There are two excitation wavelengths, $527 \mathrm{~nm}$ for the monomer form and $590 \mathrm{~nm}$ for the J-aggregate form. The magnitude of green fluorescent signals reflects the amount of damaged mitochondria. The cells were trypsinized and the cell pellets were re-suspended in $500 \mu$ of PBS, incubated with $10 \mu \mathrm{M} \mathrm{JC}-1$ for $20 \mathrm{~min}$ at $37^{\circ} \mathrm{C}$. The cells were subsequently washed once with cold PBS, suspended in a total volume of $500 \mu \mathrm{l}$, and analyzed by flow cytometry.

\section{Statistical analyses}

Data represent means \pm standard deviations. Statistical significance was determined using the one-way analysis of variance (ANOVA) with post-test Neuman-Keuls for more than two groups and Student's t test for two groups. 
Flavonoids from Orostachys Japonicus A. Berger Induce Caspase-dependent Apoptosis in U937 Human Leukemic Cells $\mathrm{p}<0.05$ was accepted as statistically significant.

\section{Results}

FEOJ induces apoptosis in U937 human leukemic cells

To investigate the anti-cancer activity of FEOJ, U937 cells were treated with various concentrations of FEOJ for $48 \mathrm{~h}$. The cell growth was assessed by MTT assay. The MTT assay revealed that the growth of U937 cells were inhibited by FEOJ treatment in a dose-dependent manner, and the $50 \%$ inhibition of cell growth (IC50) was approximately $180 \mu \mathrm{g} / \mathrm{mL}$ (Figure 1A). To determine whether the decrease in cell viability was derived from FEOJ-induced apoptosis, we measured cells with sub-G1 DNA content by flow cytometry. The cells with sub-G1 DNA content was increased in a dose-dependent (Figure $1 \mathrm{~B}$ and $1 \mathrm{C})$.

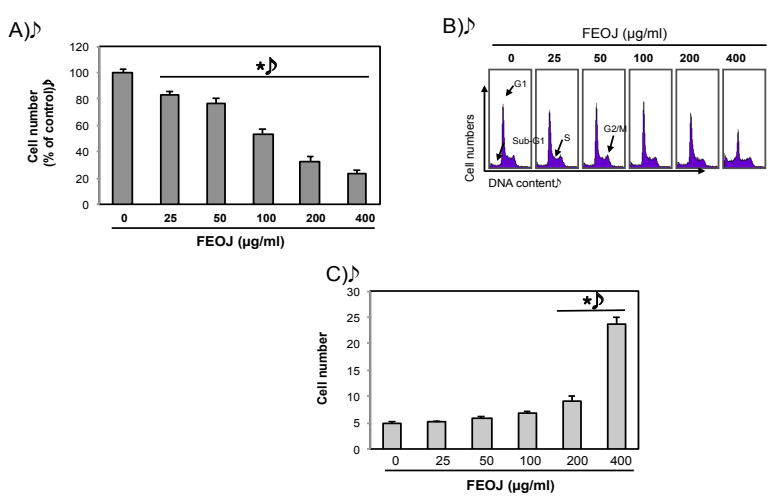

Figure 1. Effects of FEOJ on the Cell Viability of U937

Cells. The cells were seeded at the density of $1 \times 10^{5}$ cells per $\mathrm{ml}$ and incubated with the indicated concentrations of FEOJ for $48 \mathrm{~h}$ at the indicated concentrations of FEOJ. A) Cell viability was assessed by the MTT assay. Data are expressed as mean \pm SD of three independent experiments ( $* p<0.05$ versus control). B and C) By flow cytometry, the cells with sub-G1 DNA content representing the cells undergoing cell death were also analyzed.
A)s

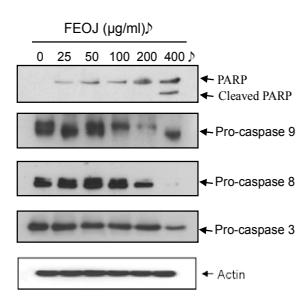

B) $)$

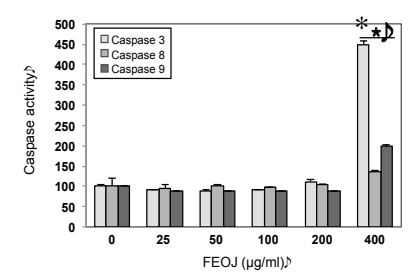

Figure 2. Activation of Caspases During the FEOJInduced Apoptosis in U937 Cells. U937 cells were Incubated at indicated concentrations of FEOJ for $48 \mathrm{~h}$. A) Equal amounts of cell lysate $(30 \mu \mathrm{g})$ were resolved by SDS-polyacrylamide gels and transferred onto nitrocellulose membranes. The membranes were probed with the anti-caspase-3, $-8,-9$ and anti-PARP antibodies. The proteins were visualized using an ECL detection system. B-actin was used as an internal control. B and C) The cell lysates from the cells treated with FEOJ for $48 \mathrm{~h}$ were assayed for in vitro caspase- $3,-8$ and -9 activity using DEVD-pNA, IETDpNA and LEHD-pNA respectively, as substrates. The released fluorescent products were measured. Each bar graph represents mean $\pm \mathrm{SD}$ of three independent experiments. ${ }^{*} \mathrm{p}<0.05$ between the treated with FEOJ and the untreated group
FEOJ-induced apoptosis is caspase-dependent in U937 human leukemic cells

Next, we investigated whether FEOJ-induced apoptosis is caspase dependent. Then we assessed the effects of FEOJ on caspases and their substrates (PARP). FEOJ decreased the expression levels of pro-caspase-3, pro-caspase- 8 , and pro-caspase- 9 in a dose-dependent manner. With the decrease of pro-caspases, the cleavages of PARP were prominent at the concentration of $400 \mu \mathrm{g} /$ $\mathrm{mL}$ of FEOJ (Figure 2A). These findings suggest that FEOJ induce apoptosis through caspase activation. To confirm this finding, we assessed proteolytic activities of caspases using colorimetric assay kits. Unfortunately, the caspase activity assay only showed that FEOJ increased proteolytic activities of caspases at the concentration of $400 \mu \mathrm{g} / \mathrm{mL}$ (Figure 2B). The caspase activity assay revealed that FEOJ increased proteolytic activities of caspases and induce caspase-dependent apoptosis.

FEOJ induces apoptosis at least partly through mitochondrial pathway and modulates Bcl-2 and IAP family members.

Mitochondria play a central role in apoptosis. As the early event of apoptosis, mitochondrial depolarization occurs. Therefore, we investigated the role of mitochondria in FEOJ-induced apoptosis by measuring MMP $(\Delta \Psi \mathrm{m})$. As shown in Figure $3 \mathrm{~A}, 48 \mathrm{~h}$-treatment of FEOJ significantly induced the loss of MMP $(\Delta \Psi \mathrm{m})$ in a dosedependent manner $(78.5 \%$ at $400 \mu \mathrm{g} / \mathrm{ml} v s 37 \%$ at the

A) $)$
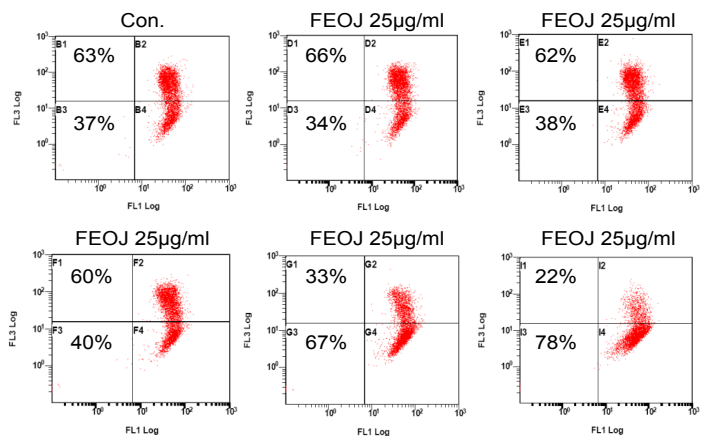

B)D

C) $)$

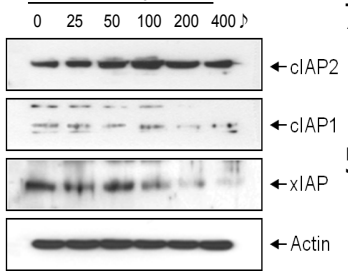

Figure 3. Effects of FEOJ on Mitochondrial Depolarization, and Bcl-2, and IAP Family Members in U937 Cells. The cells were treated with FEOJ for $48 \mathrm{~h}$ at the indicated concentrations. (A) The cells were stained with $\mathrm{JC}-1$ and incubated at $37^{\circ} \mathrm{C}$ for $20 \mathrm{~min}$. The mean $\mathrm{JC}-1$ fluorescence intensity was detected using a flow cytometer. (B and C) Equal amounts of cell lysate $(30 \mu \mathrm{g})$ were resolved by SDS-polyacrylamide gels and transferred onto nitrocellulose membranes. The membranes were probed with the indicated antibodies and detected by the ECL system. The results are from a representative experiment of at least two independent experiments that showed similar patterns 
control), indicating that FEOJ increases depolarization of the MMP $(\Delta \Psi \mathrm{m})$. The loss of MMP $(\Delta \Psi \mathrm{m})$ elicited by FEOJ lead to activation of caspase-3, which has been shown to play a pivotal role in the terminal execution phase of apoptosis, and also activate caspase 8 through the activation of Bid protein (Ashkenazi, 2002). Therefore, we checked Bid cleavages to verify the feedback pathway to the activation of caspase 8 . Western blot data showed that FEOJ induced cleavage of Bid (activation of Bid protein) in a dose-dependent manner (Figure 3B). Next, we assessed the levels of Bcl-2 and IAP family proteins which play a crucial role in apoptosis to elucidate further underlying mechanisms of FEOJ-induced apoptosis. Western blotting revealed that FEOJ suppressed Bcl-2 and XIAP (anti-apoptotic proteins) in a dose-dependent manner (Figure 3B) whereas the suppression of CIAP-1 and CIAP-2 was not observed (Figure 3C). These findings suggest that the loss of MMP $(\Delta \Psi \mathrm{m})$ and activation of Bid protein are associated with down-regulation of $\mathrm{Bcl}-2$ and X-IAP protein.

\section{FEOJ induced apoptosis at least in part by activating p38} MAPK activity.

Mitogen-activated protein kinase (MAPK) (Robinson and Cobb, 1997) and PI3K/Akt pathway (Luo et al., 2003) play important roles in regulating apoptosis, differentiation and cell death. To determine whether Mitogen-activated protein kinase (MAPK) and PI3K/ Akt pathway is associated with FEOJ-induced apoptosis, we investigated the effects of FEOJ on MAPK and Akt in U937 cells. Since the activity of MAPK and Akt is regulated by phosphorylation, we assessed the levels of phosphorylated MAPK and Akt in FEOJ-treated U937 cells. The Western blot analysis revealed that FEOJ significantly activated phosphorylation of p38 MAPK and suppressed the phosphorylation of Akt (Figure

A)S
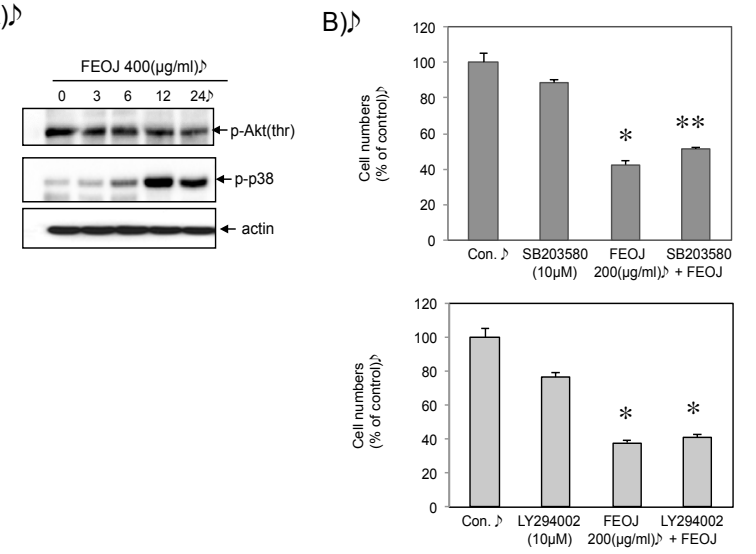

Figure 4. The Role of p38 MAPK Signaling in FEOJ-

Induced Apoptosis. The cells were seeded at the density of $1 \times 10^{5}$ cells per $\mathrm{ml}$ and incubated at the indicated concentrations of FEOJ for the indicated time duration. (A) Western blot analysis for Akt and p38 MAPK. Equal amounts of cell lysate (30 $\mu \mathrm{g})$ were resolved by SDS-polyacrylamide gels and transferred onto nitrocellulose membranes. The results are from at least two independent experiments that showed similar patterns. (B) Cell viability was assessed by the MTT assay. Data are expressed as mean \pm SD of three independent experiments $\left({ }^{*} \mathrm{p}<0.05\right.$ versus control, $* * \mathrm{p}<0.05$ versus FEOJ treatment)

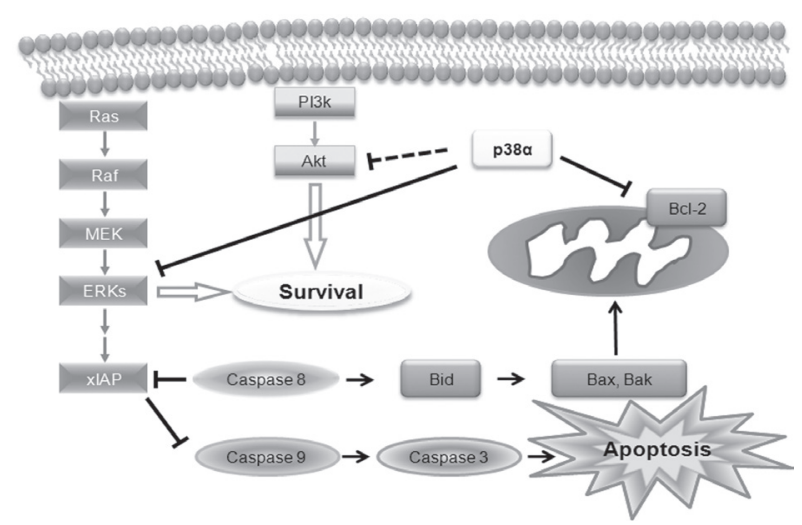

Figure 5. Schematic Representation on Apoptotic Effects of FEOJ on U937 Human Leukemic Cells. FEOJ induced apoptosis through mitochondrial pathway by inducing loss of MMP $(\Delta \Psi \mathrm{m})$, and then activates caspases. During this process, the suppression of Bcl-2 and X-IAP contributes the augmentation of the apoptosis. In addition, p38 MAPK activation may serve as one of the upstream signaling involved in FEOJ-induced apoptosis. Taken together, this study suggested that FEOJ induced caspase-dependent apoptosis through mitochondrial pathway by modulating Bcl-2 and X-IAP proteins, and that $\mathrm{p} 38 \mathrm{MAPK}$ is at least one of the important upstream signalings that regulate the apoptotic effect of FEOJ in human leukemic U937 cells

4A). To confirm this finding, we evaluated the effects of FEOJ with and without treatment of Akt inhibitor (LY294002) and p38 MAPK inhibitor (SB203580). MTT assay revealed that LY294002 and p38 MAPK inhibitor (SB203580) reduced the effect of FEOJ on cell viability (Figure 4B). Taken together, these findings suggest that FEOJ induce apoptosis at least in part by activating p38 MAPK signaling pathway.

\section{Discussion}

This study was designed to determine whether FEOJ has anti-cancer properties in human leukemia cells, and further investigate the underlying mechanisms of the apoptotic effect of FEOJ. Indeed, we demonstrated that FEOJ induced apoptosis in a dose-dependent manner in U937 human leukemic cells. The FEOJ-induced apoptosis was caspase dependent. This finding was confirmed by caspase activity assay. Many well-known phytochemicals that can safely modulate physiological function and enhance anti-cancer activity induce apoptosis through mitochondrial pathway (Chun et al., 2003; Gupta et al., 2013). Similarly, FEOJ induced apoptosis through induction of loss of MMP $(\Delta \Psi \mathrm{m})$. The loss of MMP $(\Delta \Psi \mathrm{m})$ may be related with suppression of BCl-2. Next, we assessed IAP family members which are important antiapoptotic proteins that regulate caspases. We demonstrated that FEOJ suppressed X-IAP in a dose-dependent manner, which can be associated with induction of apoptosis and activation of Bid protein. All these finding suggested that FEOJ induces caspase-dependent apoptosis at least in part through inhibiting anti-apoptotic protein expression.

When we look into the components of FEOJ, quercetin was one of the major components (Wiczkowski et al.,2008; Lee and Mitchell, 2011). Previous reports demonstrated 
Flavonoids from Orostachys Japonicus A. Berger Induce Caspase-dependent Apoptosis in U937 Human Leukemic Cells that quercetin induced apoptosis in several types of cancer cells (Psahoulia et al., 2007), and it augmented apoptosis by up-regulating DR5 expression (Jang et al., 2003). These findings are consistent with our results even though we did not demonstrate the up-regulation of DR5 by FEOJ in that FEOJ also induced caspase 8 activation. In addition, quercetin increases apoptosis by altering anti-apoptotic proteins, which supports our fining that FEOJ suppressed an anti-apoptotic factor Bcl-2, and X-IAP in U937 cells. Regarding cIAP-1, our data showed that FEOJ did not influence the expression of cIAP-1 which is consistent with previous reports showing that quercetin treatment did not affect the levels of cIAP-1 (Psahoulia et al., 2007; Kim et al., 2008). In previous studies, quercetin also leads to reduction in MMP $(\Delta \Psi \mathrm{m})$, and subsequent activation of caspases, thus ultimately inducing apoptosis via the intrinsic pathway in several types of cancer cells (Mouria et al., 2002).

Here, we also demonstrated that FEOJ-induced apoptosis is closely related to activation of p38 MAPK pathway. MAPK plays important roles in regulating apoptosis, and cell death (Robinson and Cobb, 1997), and quercetin plays a critical role in modulating cancer cell survival through p38 MAPK pathway (Chen et al., 2012). These finding support that p38 MAPK plays a critical role in FEOJ-induced apoptosis. In addition, the merit of FEOJ as compared to the quercetin is that the bioavailability of quercetin in FEOJ is much better that that quercetin aglycone (Manach et al., 2005; Gibellini et al., 2011), because quercetin in FEOJ is in the form of quercetin glucosides, which are easily absorbed in the apical membrane of enterocyte (O'Leary et al., 2003).

In summary, this study demonstrated that FEOJ induced caspase-dependent apoptosis in U937 cells. The apoptosis was triggered through mitochondrial pathway by inducing loss of $\mathrm{MMP}(\Delta \Psi \mathrm{m})$, and through modulating Bcl-2 and X-IAP proteins. In addition, FEOJ induces caspase-dependent apoptosis at least in part through the activation of p38 MAPK pathway (Figure 5). This study provides evidence that FEOJ might have anticancer property on human leukemic cells.

\section{Acknowledgements}

This work was supported by the grants from the National R \& D Program for Cancer Control, Ministry of Health \& Welfare, Republic of Korea (0820050).

\section{References}

Ashkenazi A (2002). Targeting death and decoy receptors of the tumour-necrosis factor superfamily. Nat Rev Cancer, 2, 420-30.

Chen SF, Nieh S, Jao SW, et al (2012). Quercetin suppresses drug-resistant spheres via the p38 MAPK-Hsp27 apoptotic pathway in oral cancer cells. PLoS One, 7, 49275.

Chun KH, Kosmeder JW, Sun S, et al (2003). Effects of deguelin on the phosphatidylinositol 3-kinase/Akt pathway and apoptosis in premalignant human bronchial epithelial cells. J Natl Cancer Inst, 95, 291-302.

Gibellini L, Pinti M, Nasi M, et al (2011). Quercetin and cancer chemoprevention. Evid Based Complement Alternat Med, 